\title{
Recent advances in human milk glycobiology
}

\author{
David S. Newburg ${ }^{1}$ and Gilman Grave ${ }^{2}$
}

$T^{\mathrm{T}}$ he field of human milk glycobiology is progressing rapidly, with important potential applications to health. Only a few decades ago, most experts considered human milk oligosaccharides (HMOS) to be an incidental consequence of high concentrations of glycosyltransferases (for glycoprotein and glycolipid synthesis) in proximity to high concentrations of lactose in the mammary epithelium.

Breastfeeding is known to reduce the risk of enteric and other infectious disease in infancy (1). Biological functions are now being identified for many human milk glycans, particularly the HMOS, which are glycans terminating in lactose, and the larger and more complex human milk glycoconjugates (HMGs), which are glycans attached to other noncarbohydrate scaffolds. Specific complex milk carbohydrates inhibit the adhesion of pathogens to the cell surface receptors of their target cells, an essential first step in pathogenesis. In addition, many milk glycans stimulate infant gut colonization with mutualist bacteria of the microbiota. This prebiotic feature conveys potential health benefits. Other human milk glycans modulate the mucosal immune system (2). In light of these discoveries, it is reasonable to consider HMOS as a major part of an innate immune system by which breastfeeding mothers protect their infants from disease (Figure 1).

On 21 and 22 February 2013, the Eunice Kennedy Shriver National Institute of Child Health and Human Development and the National Institute of General Medical Sciences co-sponsored a conference entitled Bioactive Glycans in Human Milk. Glycobiologists from the United States, Canada, and Europe were asked to assess the current state of our understanding of human milk glycobiology and to identify research directions that promise to advance the field (see Supplementary Table S1 online). Major limitations in our current understanding were identified as research opportunities.

One challenge is to define the concentrations of human milk glycans and their individual variation, especially that of HMOS and HMGs (3). Two factors limit this task: First is the availability of representative, well-defined, fastidiously collected milk samples that document the time of collection, time elapsed since prior feeding, representation of all milk from foremilk to hindmilk, maternal nutritional status, and method of milk expression (4). Second is the need for analytical methods to measure HMOS and HMGs that fully resolve the HMOS from lactose and from each other; that provide a linear response

over the wide variation of HMOS concentrations in milk, with a low coefficient of variation (precision) and high and consistent recovery from sample (accuracy); that generate results independent of the milk matrix; and that have relatively high throughput. Most importantly, the combined error (precision + accuracy) must be lower than the biological differences to be measured among milk samples. Liquid chromatography with tandem mass spectrometry, with proper stationary and mobile phases and scrupulous validation, fulfills these criteria (5-7).

Expression of individual milk components differs among humans $(8,9)$, and human milk varies with stage of lactation, diet, and other biological parameters. HMOS expression is especially heterogenous (10), with maternal glycosyltransferase genotypes-notably the fucosyltransferases-being a major factor driving variation among individuals and populations. For example, a fucosylated HMOS that inhibits a specific pathogen's binding to its host target requires a particular fucosyltransferase for its synthesis. A mother with a null mutation in the gene encoding the required fucosyltransferase produces milk lacking that fucosylated HMOS, and cannot protect her infant from that specific pathogen (11). Thus, heterogeneity of glycan expression in milk implies highly variable protection from disease. However, milks that protect poorly against one pathogen may protect more strongly against another, depending on the overall HMOS expression pattern.

The totality of protection by human milk involves a cocktail of hundreds of glycans that vary widely across individuals and populations. Multifactorial synergy of structural moieties in their monovalent, polyvalent (single molecule containing multiples of one glycan moiety), and multivalent (single molecule containing multiple types of glycan moieties) forms should be investigated. No single composition is likely to be appropriate at all times in all groups. Beyond protection of the individual infant by its own mother's milk, heterogeneous expression of HMOS patterns might provide a collective survival advantage through herd immunity, in which incomplete individual protection is still adequate to break the chain of transmission of infection across exposed individuals, thereby protecting the group. Thus, therapeutic agents that may be developed from research on the glycobiology of human milk should be tailored to account for the predominant glycan synthesis gene frequencies in specific populations, the endemic microbial pathogens, and the specific public health objectives.

\footnotetext{
'Department of Biology, Boston College, Chestnut Hill, Massachusetts; ${ }^{2}$ Pediatric Growth and Nutrition Branch, Eunice Kennedy Shriver National Institute of Child Health and
} Human Development, National Institutes of Health, Bethesda, Maryland. Correspondence: David S. Newburg (david.newburg@bc.edu) 


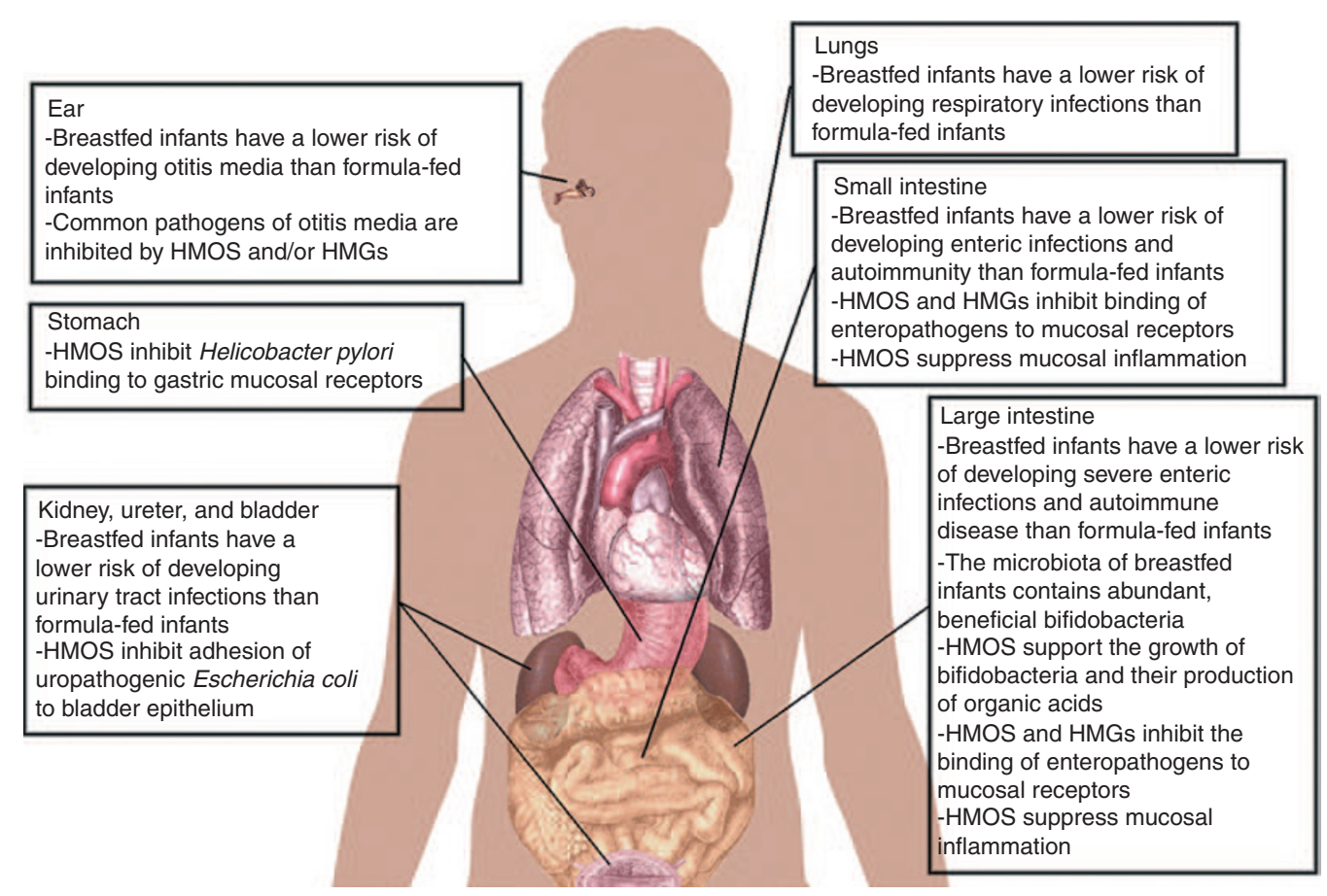

Figure 1. Sites of human milk glycan activity. The primary site for human milk oligosaccharides (HMOS) activity is the lumen of the gut, as the preponderance of HMOS are not digested or absorbed. However, those HMOS that are absorbed are excreted into the urine, and there they could inhibit uropathogenic organisms. The mechanisms whereby orally ingested HMOS affect sites distant from the gut are unknown, but can be speculated to be a direct effect of HMOS in the bloodstream, modulation of the global mucosal immune system through a primary effect on intestinal mucosal immune status, or mediated through secondary metabolites of HMOS fermentation in the gut that traverse the bloodstream. HMGs, human milk glycoconjugates.

Oligosaccharides were originally defined as glycans containing between two and ten sugars, and the major oligosaccharides originally isolated from milk fell within this range. However, HMOS are now best defined by their specific structural features (12), including lactose at the reducing end, a backbone of alternating $\mathrm{N}$-acetyl glucosamine and galactose, sporadic branching by $\mathrm{N}$-acetyl glucosamine $\beta 1,6$-galactose linkages, and often terminal decoration by fucose, sialic acid, or both. The two major HMOS families are distinguished by the presence of sialic acid; acidic HMOS contain sialic acid, while neutral HMOS do not (Figure 2).

Variable expression of fucosyltransferase genes, notably FUT2 (encoding an 1,2 fucosyltransferase) and FUT3 (encoding the $\alpha 1,4$ fucosyltransferase), underlie the various major Lewis histoblood group antigens found on erythrocytes and other tissues, especially intestinal mucosa. It is interesting to note that each of these Lewis motifs corresponds to a homologous major neutral HMOS, including lacto- $N$-fucopentaose I ( $\mathrm{H}-1$, Lewis d), 2'-fucosyllactose ( $\mathrm{H}-2)$, lacto- $\mathrm{N}$-fucopentaose II (Lewis a), lacto- $N$-fucohexaose I (Lewis b), lacto- $N$-tetraose (Lewis c), 3-fucosyllactose and lacto- $\mathrm{N}$-fucopentaose III, (Lewis $\mathrm{x}$ ), and lactodifucotetraose (Lewis y). Likewise, sialylated Lewis histoblood group antigens have homologous sialylated fucosyloligosaccharides in milk (13). Other common sialylated motifs in human tissues have corresponding acidic (sialyl-) oligosaccharides in milk. Thus, each of the major milk oligosaccharides seems to correspond to a specific chemical niche found in the intestinal mucosa; multivalent HMGs contribute additional complexity and richness to this hypothesis. Continued characterization of the vast structural diversity of HMOS and HMGs, both within individuals and among populations, will enable further evaluation of their structure-function relationships (14).

Defining the biological activities of carbohydrate components of human milk requires in vitro and in vivo model systems; the glycobiology of these models must match the phenomenon under investigation. For example, processes mediated through a fucosylated structure require a model that expresses the relevant fucosylated structure. Thus, use of the popular rodent model should be carefully evaluated for each phenomenon and, when appropriate, other models, including porcine models (15) and ex vivo human intestine, should be considered.

Such models have produced strong evidence for the inhibition of pathogen binding by HMOS, including Campylobacter jejuni, Escherichia coli, and Salmonella. HMGs inhibit infection by Entamoeba histolyticia (16), rotavirus, norovirus, and HIV.

The heterogeneity of HMOS expression in conjunction with the fastidious specificity of many pathogens suggests highly specific functions for the hundreds, and possibly thousands, of individual HMOS (17) and HMGs. Inhibition of individual pathogens can reside in fucosylated, sialylated, or sialylated fucosylated HMOS, or the larger polyvalent and multivalent HMG $(14,18,19)$. Individual HMOS and HMGs may also function synergistically. These considerations suggest that mixtures of HMOS and HMGs may have wider utility than components used singly. A library of pure HMOS and HMGs would allow this putative synergy to be tested systematically. 


\section{NIH workshop on bioactive milk glycans $\quad$ Special Article}
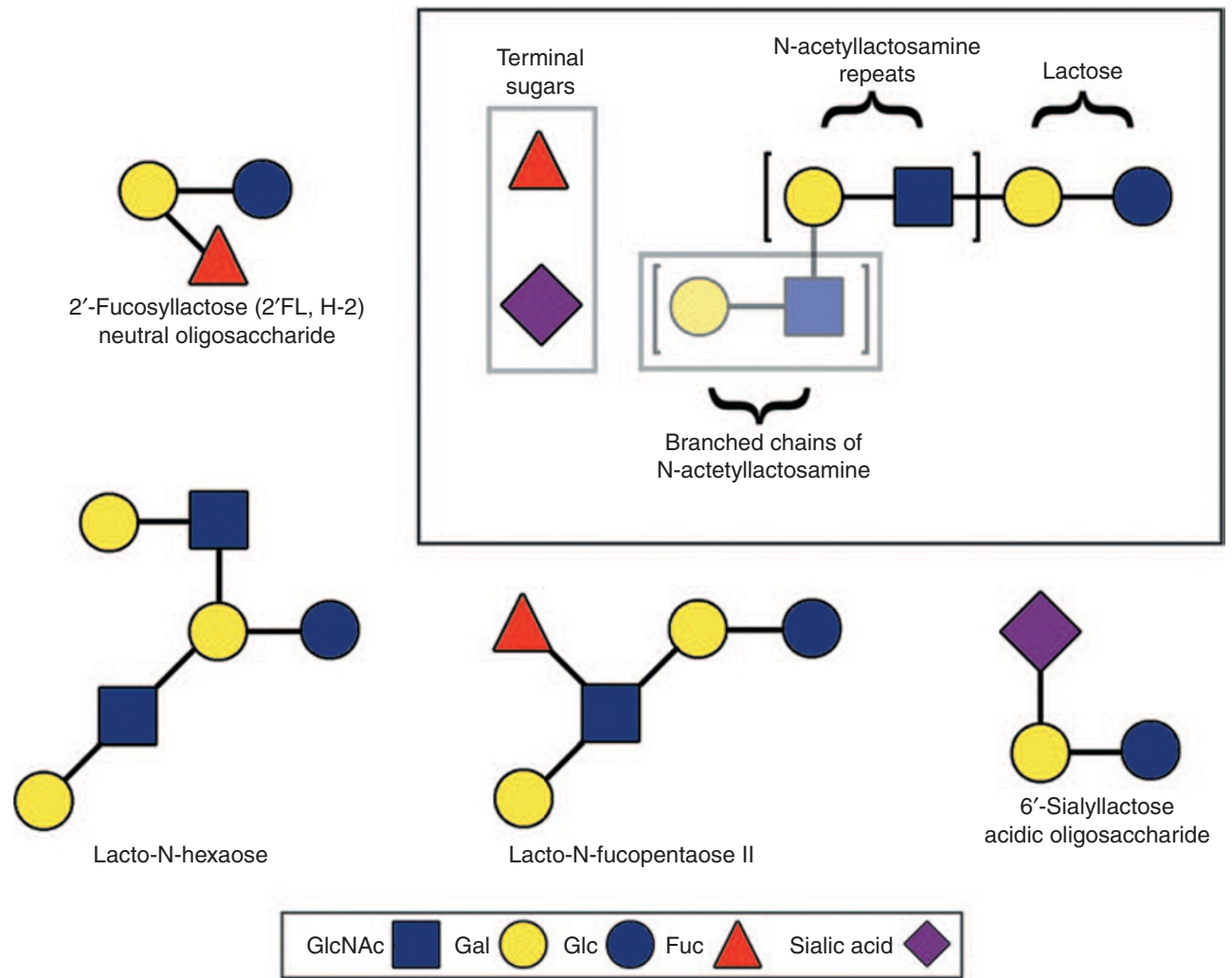

Figure 2. Human milk oligosaccharides (HMOS). The human milk oligosaccharides contain lactose on the reducing end, and most contain a polylactosamine backbone. In contrast to bovine milk, human milk oligosaccharides are a major component, with the majority being fucosylated. The acidic oligosaccharides contain sialic acid. Fuc, fucose; Gal, galactose; Glc, glucose; GlcNAc, N-acetylglucosamine; sialic acid, N-acetylneuraminic acid (some N-glycolylneuraminic acid).

Another mechanism by which HMOS protect infants against pathogens is through prebiotic effects. Prebiotics are indigestible dietary glycans that confer health benefits by modifying the intestinal microbiota. They are defined by their carbohydrate nature, oral ingestion, minimal digestion or absorption in the proximal gastrointestinal tract (20), and selective fermentation by beneficial bacteria in the distal gut. HMOS cause an increase in bifidobacteria in the infant gut microbial community; they are fermented by microbiota to produce organic acids that reduce ambient $\mathrm{pH}$. Thus, HMOS are considered to be prebiotics. Supplementing formula with lactose-derived and plant-derived prebiotics, GOS-FOS (a mixture of galactosyloligosaccharides and fructosyloligosaccharides), increases colonization of the infant gut by bifidobacteria and increases fermentation that acidifies the gut. Although many pathogens are inhibited by gut acidification that is produced by GOSFOS (21) and HMOS alike, there are, undoubtedly, many other commonalities and differences in their effects $(22,23)$.

Human milk contains a large number of molecules larger than HMOS, the majority of which are glycosylated. These large glycans are also highly bioactive. Many HMGs competitively inhibit pathogen binding. Moreover, many of the antibodies, hormones, and other signaling agents of human milk are glycosylated. Isolation and full chemical definition of these molecules is daunting, and defining their activities and mechanism of action (24) remains in its infancy. HMGs include glycosaminoglycans (25), mucins, glycoproteins, glycopeptides, and glycolipids (Figure 3 ).

Glycosaminoglycans and mucins are the largest in size, and remain the most poorly defined, but are highly intriguing. For example, hyaluronic acid promotes antimicrobial peptide expression in intestinal mucosa that may contribute to maintenance of epithelial integrity (26). Defining the active form of this glycosaminoglycan molecule and comparing it with milk hyaluronic acid could be the basis for a prophylaxis or therapy against chronic intestinal bowel diseases. Other human milk glycosaminoglycans inhibit pathogens, including HIV. Technologies are emerging for synthesis of multi- and polyvalent molecules and portions of HMGs in quantities that could support ex vivo and in vivo testing. The hypothesis that monovalent HMOS have broad specificity, while the larger HMG molecules have greater affinity and more specificity, could be directly tested as these molecules become available. Also, the availability of pure HMGs would clarify specific mechanisms of action through the evaluation of binding kinetics and related techniques (27).

Translating current and emerging research findings into practical prevention and treatment of disease in infants will require a sustained research effort. Binding and inhibition of binding to glycan receptors by pathogen ligands can be screened on glycan arrays. Kinetics of binding can be investigated by measuring binding and binding inhibition through 

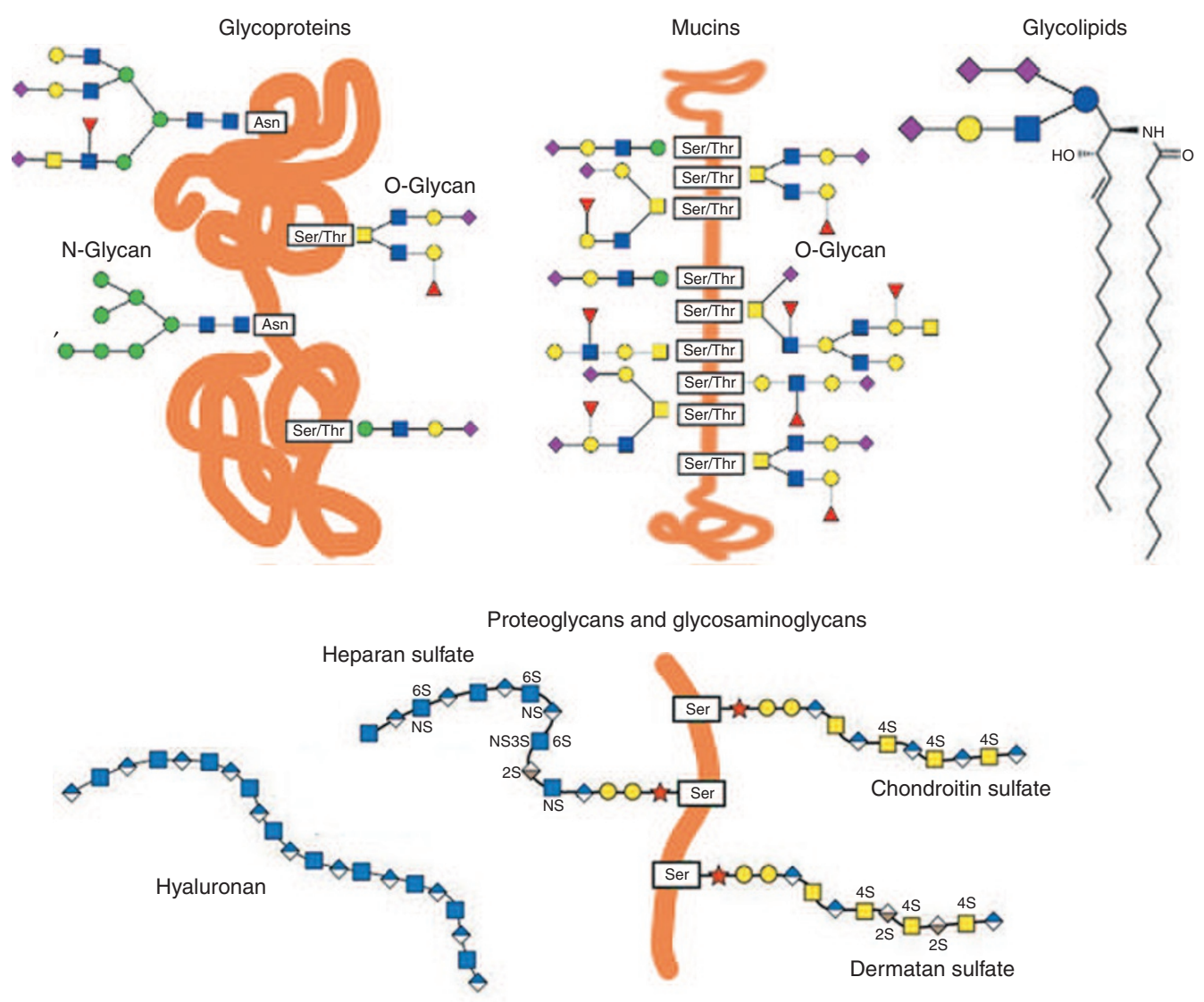

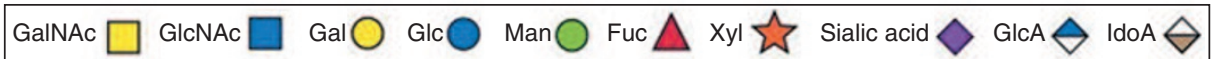

Figure 3. Human milk glycoconjugates (HMGs). Most of the major macromolecules of human milk are glycosylated, many heavily. The principal families of glycoconjugates in milk include the mucins, glycosaminoglycans, glycoproteins, glycopeptides, and glycolipids, with examples depicted in this figure. Fuc, fucose; Gal, galactose; GalNAc, N-acetylgalactosamine; Glc, glucose; GlcA, glucuronic acid; GlcNAc, N-acetylglucosamine; IdoA, iduronic acid; Man, mannose; sialic acid, $\mathrm{N}$-acetylneuraminic acid (some N-glycolylneuraminic acid); Xyl, xylose.

changes in physical surface characteristics when glycanlinked surfaces are bound, such as the change in refractive index induced by glycan binding in surface plasmon resonance. Animal models for testing activities of HMOS and HMGs can be extended and validated to ensure that the relevant glycan receptors and biological pathways are represented. The diversity of glycan expression in humans and multiple specificities in strains of pathogens must be taken into account, and may require developing animal models using multiple species, strains, and genetic modifications. Ideally, when a biologic activity of HMOS or HMGs is discovered in these models, it should be confirmed in human tissue ex vivo, if possible, before going on to human trials. Potential synergy between HMOS and HMGs can be investigated in these models. One strategy is to investigate human milk fractions for activities, and if multiple fractions are active, or if activity is lost during fractionation, the fractions can be tested in combination. Similarly, pure glycans of natural or synthetic origin can be tested individually and in combination to investigate potential synergy. To utilize these models most effectively, research in human milk glycobiology could be expedited through development and dissemination of other new tools $(28,29)$, including libraries of synthetic HMOS (30) and HMGs, and public access to stored databases with informatics support. Communal resources could be developed that provide for quality control, open distribution, and sharing among participants. A complete library of HMOS at a milligram scale might be the logical first step (31). Creation of a more complete library of HMOS on a kilogram scale, ultimately to good manufacturing practice standards, would facilitate in vivo and clinical studies. HMOS availability would also stimulate synthesis of HMGs. Federal funding has supported much of the basic research performed to date, while industry has supported translational research in this area. Translational research requires material manufactured to good manufacturing practice standards, and includes preclinical research, human trials, and patent protection. However, individual HMOS or HMGs tested by industry ideally could be made available for ancillary basic research, for example, testing in combination with other HMOS or testing in polyvalent and multivalent forms. Accelerating research on the benefits of dietary HMOS and HMGs could represent a significant advance in promoting health in populations at high risk of enteric disease. 
SUPPLEMENTARY MATERIAL

Supplementary material is linked to the online version of the paper at http:// www.nature.com/pr

\section{ACKNOWLEDGMENTS}

The authors acknowledge the support, help, and insight provided by Sarah Glavin and Rosalina Bray of the National Institute of Child Health and Human Development, Pamela Marino of the National Institute of General Medical Sciences, Terry Taylor and Michaela Oakley of Palladian Partners, and David Hill of Boston College.

\section{STATEMENT OF FINANCIAL SUPPORT}

Supported in part by grants HD059140, HD013021, AI075563, and HD061930 by the National Institutes of Health, Bethesda, MD.

Disclosure: D.S.N. owns a financial interest in Glycosyn, LLC, which synthesizes human milk oligosaccharides. The company could benefit by reports of HMOS bioactivity. Boston College manages this conflict of interest.

\section{REFERENCES}

1. Ip S, Chung M, Raman G, et al. Breastfeeding and maternal and infant health outcomes in developed countries. Evid Rep Technol Assess (Full Rep) 2007;153:1-186.

2. Jantscher-Krenn E, Zherebtsov M, Nissan C, et al. The human milk oligosaccharide disialyllacto-N-tetraose prevents necrotising enterocolitis in neonatal rats. Gut 2012;61:1417-25.

3. Newburg DS, Neubauer SH. Carbohydrates in milks: analysis, quantities, and significance. In: Jensen RG, ed. Handbook of Milk Composition. San Diego, CA: Academic Press, 1995:273-349.

4. Geraghty SR, Davidson BS, Warner BB, et al. The development of a research human milk bank. J Hum Lact 2005;21:59-66.

5. Bao Y, Chen C, Newburg DS. Quantification of neutral human milk oligosaccharides by graphitic carbon high-performance liquid chromatography with tandem mass spectrometry. Anal Biochem 2013;433:28-35.

6. Wu S, Salcedo J, Tang N, et al. Employment of tandem mass spectrometry for the accurate and specific identification of oligosaccharide structures. Anal Chem 2012;84:7456-62.

7. Nwosu CC, Aldredge DL, Lee H, et al. Comparison of the human and bovine milk N-glycome via high-performance microfluidic chip liquid chromatography and tandem mass spectrometry. J Proteome Res 2012;11:2912-24.

8. Thurl S, Munzert M, Henker J, et al. Variation of human milk oligosaccharides in relation to milk groups and lactational periods. Br J Nutr 2010;104:1261-71.

9. Coppa GV, Gabrielli O, Zampini L, et al. Oligosaccharides in 4 different milk groups, Bifidobacteria, and Ruminococcus obeum. J Pediatr Gastroenterol Nutr 2011;53:80-7.

10. Erney RM, Malone WT, Skelding MB, et al. Variability of human milk neutral oligosaccharides in a diverse population. J Pediatr Gastroenterol Nutr 2000;30:181-92.

11. Totten SM, Zivkovic AM, Wu S, et al. Comprehensive profiles of human milk oligosaccharides yield highly sensitive and specific markers for determining secretor status in lactating mothers. J Proteome Res 2012;11:6124-33.

12. McNaught AD. International Union of Pure and Applied Chemistry and International Union of Biochemistry and Molecular Biology. Joint Commission on Biochemical Nomenclature. Nomenclature of carbohydrates. Carbohydr Res 1997;297:1-92.
13. Blank D, Gebhardt S, Maass K, et al. High-throughput mass finger printing and Lewis blood group assignment of human milk oligosaccharides. Anal Bioanal Chem 2011;401:2495-510.

14. Yu Y, Mishra S, Song X, et al. Functional glycomic analysis of human milk glycans reveals the presence of virus receptors and embryonic stem cell biomarkers. J Biol Chem 2012;287:44784-99.

15. Hester SN, Chen X, Li M, et al. Human milk oligosaccharides inhibit rotavirus infectivity in vitro and in acutely infected piglets. Br J Nutr 2013;110:1233-42.

16. Jantscher-Krenn E, Lauwaet T, Bliss LA, Reed SL, Gillin FD, Bode L. Human milk oligosaccharides reduce Entamoeba histolytica attachment and cytotoxicity in vitro. Br J Nutr 2012;108:1839-46.

17. Stahl B, Thurl S, Zeng J, et al. Oligosaccharides from human milk as revealed by matrix-assisted laser desorption/ionization mass spectrometry. Anal Biochem 1994;223:218-26.

18. El-Hawiet A, Kitova EN, Kitov PI, et al. Binding of Clostridium difficile toxins to human milk oligosaccharides. Glycobiology 2011;21:1217-27.

19. Newburg DS, Ruiz-Palacios GM, Altaye M, et al. Innate protection conferred by fucosylated oligosaccharides of human milk against diarrhea in breastfed infants. Glycobiology 2004;14:253-63.

20. Albrecht S, Schols HA, van Zoeren D, et al. Oligosaccharides in feces of breast- and formula-fed babies. Carbohydr Res 2011;346:2173-81.

21. Arslanoglu S, Moro GE, Boehm G. Early supplementation of prebiotic oligosaccharides protects formula-fed infants against infections during the first 6 months of life. J Nutr 2007;137:2420-4.

22. Arslanoglu S, Moro GE, Boehm G, Wienz F, Stahl B, Bertino E. Early neutral prebiotic oligosaccharide supplementation reduces the incidence of some allergic manifestations in the first 5 years of life. J Biol Regul Homeost Agents 2012;26(3 Suppl):49-59.

23. Fuhrer A, Sprenger N, Kurakevich E, Borsig L, Chassard C, Hennet T. Milk sialyllactose influences colitis in mice through selective intestinal bacterial colonization. J Exp Med 2010;207:2843-54.

24. Nanthakumar NN, Meng D, Newburg DS. Glucocorticoids and microbiota regulate ontogeny of intestinal fucosyltransferase 2 requisite for gut homeostasis. Glycobiology 2013;23:1131-41.

25. Coppa GV, Gabrielli O, Buzzega D, et al. Composition and structure elucidation of human milk glycosaminoglycans. Glycobiology 2011;21:295303.

26. Hill DR, Kessler SP, Rho HK, Cowman MK, de la Motte CA. Specific-sized hyaluronan fragments promote expression of human $B$-defensin 2 in intestinal epithelium. J Biol Chem 2012;287:30610-24.

27. El-Hawiet A, Shoemaker GK, Daneshfar R, Kitova EN, Klassen JS. Applications of a catch and release electrospray ionization mass spectrometry assay for carbohydrate library screening. Anal Chem 2012;84:50-8.

28. Smith DF, Cummings RD. Application of microarrays for deciphering the structure and function of the human glycome. Mol Cell Proteomics 2013;12:902-12.

29. Ko KS, Jaipuri FA, Pohl NL. Fluorous-based carbohydrate microarrays. J Am Chem Soc 2005;127:13162-3.

30. Sugiarto G, Lau K, Qu J, et al. A sialyltransferase mutant with decreased donor hydrolysis and reduced sialidase activities for directly sialylating LewisX. ACS Chem Biol 2012;7:1232-40.

31. Wu S, Tao N, German JB, Grimm R, Lebrilla CB. Development of an annotated library of neutral human milk oligosaccharides. J Proteome Res 2010;9:4138-51. 